\title{
A NEW MULTI-FIBER MODEL FOR LOW ANGULAR RESOLUTION DIFFUSION MRI
}

\author{
Aymeric Stamm $^{\star} \quad$ Patrick Pérez $\quad$ Christian Barillot ${ }^{\star}$ \\ * Visages INSERM/INRIA U746, IRISA - UMR CNRS 6074, Rennes, France \\ $\dagger$ Technicolor, Rennes, France
}

\begin{abstract}
Diffusion MRI is a tool of choice for the analysis of the brain white matter fiber pathways. When translated to clinics, the short acquisition time leads to low angular resolution diffusion (LARD) images. Fiber pathways are then inferred assuming Gaussian diffusion (a.k.a. DTI) that provides one fiber orientation per voxel. In the past decade, recent researches highlight more intricate intra-voxel fiber configurations using higher angular resolution diffusion images.

In this paper, we introduce a novel non-Gaussian parametric modeling of the diffusion that accounts for multiple intravoxel fiber orientations, while being compatible with LARD acquisitions. It can indeed be estimated from standard clinical diffusion images with a crossing angle resolution of $30^{\circ}$.
\end{abstract}

Index Terms - diffusion MRI, non-Gaussian parametric EAP, crossing fibers.

\section{INTRODUCTION}

A widespread application of diffusion magnetic resonance imaging (dMRI) is the study of the brain white matter fiber pathways into which diffusion is restricted along the neuronal pathways. Learning the distribution of the induced random motion, known as the ensemble average propagator (EAP), thus allows the inference of fibers geometry.

In clinical brain imaging, dMRI sequences usually consist in acquiring $n_{g} \leq 30$ diffusion-weighted (DW) images resulting from the application of $n_{g}$ magnetic field gradients with common low intensity encoded via the $b$-value $(b \leq$ $\left.1500 \mathrm{~s} / \mathrm{mm}^{2}\right)$ and $n_{g}$ different directions $\left\{\boldsymbol{g}_{i}\right\}_{i \in \llbracket 1, n_{g} \rrbracket}$. To the best of our knowledge, these low angular resolution diffusion (LARD) images are analyzed in clinics only through diffusion tensor imaging (DTI) [1], which relies on the assumption that the EAP follows a centered 3D Gaussian distribution with covariance matrix proportional to the diffusion tensor. This model allows one to determine a single fiber orientation (FO) per voxel. Yet, in research context, higher angular resolution samplings $\left(n_{g} \geq 60\right)$ [2], including multiple $b$-values $[3,4,5]$, have revealed multiple intra-voxel FO.

In this work, we introduce a non-Gaussian parametric modeling of the EAP that accounts for multiple intra-voxel
FOs while being compatible with classical clinical dMRI sequences. The parametric distribution is presented in Section 2.1 and its estimation from DW images is outlined in Section 2.2. Validation on both synthetic and real data is given in Section 3, showing in particular the ability of the model to estimate crossing fibers from LARD images.

\section{THEORY}

\subsection{Parametric modeling of the EAP}

In the same vein as DTI [1], the displacements of water molecules in a given voxel are represented by a random variable $\mathbf{x}=\sqrt{2 \tau} \mathbf{y}$, where $\tau$ is the diffusion time between two successive magnetic field gradients, and the random variable $\mathbf{y}$ is modeled hereafter.

Single-compartment model. Let us first consider a single FO $\pm \boldsymbol{\mu}$ per voxel, $\|\boldsymbol{\mu}\|=1$. The displacements of water molecules along direction ${ }^{1} \boldsymbol{\mu}$ are represented by a random variable $\mathbf{w}$ which is modeled as the sum of two independent random variables, $\mathbf{w}=\mathbf{v}+\mathbf{z}$, where:

- $\mathbf{v}$ follows a von Mises \& Fisher probability distribution on the 2-dimensional sphere of radius $R>0$ with mean direction $\boldsymbol{\mu}$ and concentration parameter $\kappa \geq 0$. The higher the concentration parameter, the more likely water molecules are to diffuse along direction $\boldsymbol{\mu}$; when it is nil, the direction of molecular displacements is uniformly distributed over the sphere; $\boldsymbol{\mu}$ can thus be interpreted as the direction of diffusion, whereas $\kappa$ can be interpreted as a measure of anisotropy of the diffusion. The von Mises \& Fisher probability distribution admits a pdf on the 2-dimensional sphere of radius $R$ which is given by ${ }^{2}$ :

$$
f_{\mathbf{v}}(\boldsymbol{v} ; \boldsymbol{\mu}, \kappa, R)=\kappa\left(4 \pi R^{3} \sinh \kappa\right)^{-1} \exp \left\{\kappa R^{-1} \boldsymbol{\mu}^{\prime} \boldsymbol{v}\right\}
$$

for any $\boldsymbol{v} \in \mathbb{R}^{3}$ such that $\|\boldsymbol{v}\|=R$.

- $\mathbf{z}$ follows a centered Gaussian probability distribution defined on $\mathbb{R}^{3}$ and parametrized by a cylindrically constrained covariance matrix $D$, akin to the diffusion tensor, completely determined by the ratio, set to $\kappa+1$, of its largest non-zero eigenvalue to its smallest non-zero eigenvalue and the value

\footnotetext{
${ }^{1}$ An orientation $\pm \boldsymbol{\mu}$ is characterized by two opposite directions $\boldsymbol{\mu}$ and $-\boldsymbol{\mu}$

${ }^{2}$ To distinguish a random variable from one realization of it, we adopt sanserif and curvilinear types to designate the former and the latter respectively.
} 
of its largest eigenvalue set to $R^{2}$ with associated eigenvector set to $\boldsymbol{\mu}$. Hence, $D=R^{2}(\kappa+1)^{-1}\left(I_{3}+\kappa \boldsymbol{\mu} \boldsymbol{\mu}^{\prime}\right)$, where $I_{3}$ is the $3 \times 3$ identity matrix. If $\kappa \rightarrow+\infty$, then $D=R^{2} \boldsymbol{\mu} \boldsymbol{\mu}^{\prime}$ and thus $R$ represents the mean radial displacement along the direction of diffusion $\mu$; if $\kappa \rightarrow 0$, then $D=R^{2} I_{3}$ and thus $R$ represents the mean radial displacement. The centered "cylindrical" Gaussian pdf reads:

$$
\begin{gathered}
f_{\mathbf{z}}(\boldsymbol{z} ; \boldsymbol{\mu}, \kappa, R)=(\kappa+1)(R \sqrt{2 \pi})^{-3} \\
\times \exp \left\{-\frac{(\kappa+1)\|\boldsymbol{z}\|^{2}-\kappa\left(\boldsymbol{\mu}^{\prime} \boldsymbol{z}\right)^{2}}{2 R^{2}}\right\}, \text { for any } \boldsymbol{z} \in \mathbb{R}^{3} .
\end{gathered}
$$

- $\mathbf{v}$ and $\mathbf{z}$ are statistically independent.

The pdf of the random variable $w$ then amounts to a convolution of the von Mises \& Fisher pdf (1) and the Gaussian pdf (2). It is a 4-parameter pdf given by:

$$
\begin{array}{r}
f_{\mathbf{w}}(\boldsymbol{w} ; \boldsymbol{\mu}, \kappa, R)=C \exp \left\{-\frac{(\kappa+1) w_{\perp}^{2}+w_{\| /}^{2}}{2}\right\} \\
\times \int_{-1}^{1} \exp \left\{\frac{\kappa}{2} t^{2}+\left(\kappa+w_{/ /}\right) t\right\} I_{0}\left((\kappa+1) w_{\perp} \sqrt{1-t^{2}}\right) \mathrm{d} t,
\end{array}
$$

for any $\boldsymbol{w} \in \mathbb{R}^{3}$, where $I_{0}$ is the zero-th order modified Bessel function and

$$
\begin{aligned}
\left(w_{\|}, w_{\perp}\right) & :=R^{-1}\left(\boldsymbol{\mu}^{\prime} \boldsymbol{w}, \sqrt{\|\boldsymbol{w}\|^{2}-\left(\boldsymbol{\mu}^{\prime} \boldsymbol{w}\right)^{2}}\right), \\
C & :=\frac{\kappa(\kappa+1) \sqrt{2}}{8 \pi^{3 / 2} R^{3} \sinh \kappa} \exp \left\{-\frac{\kappa+1}{2}\right\} .
\end{aligned}
$$

Finally, in a fiber pathway with $\mathrm{FO} \pm \boldsymbol{\mu}$, water molecules are assumed to diffuse along directions $\boldsymbol{\mu}$ and $-\boldsymbol{\mu}$ in equal proportions so that the pdf of $\mathbf{y}$ is given by:

$$
f_{\mathbf{y}}(\boldsymbol{y} ; \pm \boldsymbol{\mu}, \kappa, R)=\frac{1}{2} f_{\mathbf{w}}(\boldsymbol{y} ; \boldsymbol{\mu}, \kappa, R)+\frac{1}{2} f_{\mathbf{w}}(\boldsymbol{y} ;-\boldsymbol{\mu}, \kappa, R) .
$$

Multi-compartment model. Assuming $M$ putative FO $\left\{ \pm \boldsymbol{\mu}_{i}\right\}_{i=1, \ldots, M}$ per voxel, the pdf of $\mathbf{y}$ is modeled as a mixture of $M+1$ pdfs $f_{0}, f_{1}, \ldots, f_{M}$ as pioneered in [2]:

- $f_{0}$ characterizes water molecules subject to isotropic diffusion; it is a 1-parameter pdf given by Eq.(4) with $\kappa=0$ and parameter $R_{0}>0$; its associated weight $a_{0} \in[0,1]$ is a parameter of the model;

- for any $i \in \llbracket 1, M \rrbracket, f_{i}$ characterizes the diffusion along FO $\pm \boldsymbol{\mu}_{i}$; it is a 4-parameter pdf given by Eq.(4) with parameters $\left( \pm \boldsymbol{\mu}_{i}, \kappa_{i}, R_{i}\right),\left\|\boldsymbol{\mu}_{i}\right\|=1, \kappa_{i} \geq 0$ and $R_{i}>0$; its associated weight $a_{i}$ is set proportional to $\kappa_{i}$.

The covariance matrices $D_{i}$ involved in each compartment are akin to diffusion tensors. The largest and smallest eigenvalues, $R_{i}^{2}$ and $R_{i}^{2} /\left(\kappa_{i}+1\right)$ respectively, can thus be interpreted as the principal and transverse diffusivities of each compartment, respectively. Based on the argument that nerve fibers share similar geometries, these quantities are often assumed identical in each compartment $[6,7]$. We follow the same lines for the transverse diffusivity but we let each compartment have its own principal diffusivity to robustify the estimation of the associated FO. For any $i \in \llbracket 1, M \rrbracket$, we set $R_{i}^{2}=\left(\kappa_{i}+1\right) \lambda$, where $\lambda>0$ is the transverse diffusivity.
In our multi compartment model, the EAP thus amounts to:

$$
\begin{aligned}
& f_{\mathbf{y}}\left(\boldsymbol{y} ;\left\{ \pm \boldsymbol{\mu}_{i}, \kappa_{i}\right\}_{i \in \llbracket 1, M \rrbracket}, \lambda, a_{0}\right)=a_{0} f_{0}(\boldsymbol{y} ; \sqrt{\lambda}) \\
& +\frac{1-a_{0}}{\sum_{\ell=1}^{M} \kappa_{\ell}} \sum_{i=1}^{M} \kappa_{i} f_{i}\left(\boldsymbol{y} ; \pm \boldsymbol{\mu}_{i}, \kappa_{i}, \sqrt{\left(\kappa_{i}+1\right) \lambda}\right) .
\end{aligned}
$$

It is thus parametrized by $3 M+2$ parameters (e.g., 5 parameters for a single fiber, 8 for crossing fibers), namely:

- the spherical coordinates $\left(\theta_{i}, \phi_{i}\right) \in[0, \pi] \times[0,2 \pi[$ of the putative fiber orientation $\pm \boldsymbol{\mu}_{i}$, for any $i \in \llbracket 1, M \rrbracket$;

- the concentration of water molecules $\kappa_{i} \geq 0$ around the putative fiber orientation $\pm \boldsymbol{\mu}_{i}$, for any $i \in \llbracket 1, M \rrbracket$;

- the transverse diffusivity $\lambda>0$, identical for each FO;

- the proportion $a_{0} \in[0,1]$ of water molecules that are subject to isotropic diffusion.

\subsection{Estimation of the EAP from noisy DW images}

The theoretical DW intensity $A(b, \boldsymbol{g})$ in each voxel of a DW image depends on the intensity $(b)$ and direction $(g)$ of the corresponding magnetic field gradient and is the Fourier transform (FT) of the EAP [8]:

$$
\frac{A(b, \boldsymbol{g})}{A(\mathbf{0})}=\mathrm{FT}\left[f_{\mathbf{x}}\right]\left(\sqrt{\frac{b}{\tau}} \boldsymbol{g}\right)=\mathrm{FT}\left[f_{\mathbf{y}}\right](\sqrt{2 b} \boldsymbol{g}),
$$

where $A(\mathbf{0})$ is the theoretical DW intensity in absence of magnetic field gradient.

Assuming that the pdf of $\mathbf{y}$ is given by Eq.(5), we get:

$$
\begin{array}{r}
\frac{A\left(b, \boldsymbol{g} ;\left\{ \pm \boldsymbol{\mu}_{i}, \kappa_{i}\right\}_{i \leq M}, \lambda, a_{0}\right)}{A(\mathbf{0})}=a_{0} \operatorname{FT}\left[f_{0}\right](\sqrt{2 b} \boldsymbol{g}) \\
+\frac{1-a_{0}}{\sum_{\ell=1}^{M} \kappa_{\ell}} \sum_{i=1}^{M} \kappa_{i} \operatorname{FT}\left[f_{i}\right](\sqrt{2 b} \boldsymbol{g}),
\end{array}
$$

where the FT of $f_{\mathbf{y}}$ modeled by Eq.(4) is given by [9]:

$$
\begin{gathered}
\operatorname{FT}\left[f_{\mathbf{y}}\right](\boldsymbol{t})=\exp \left\{-\frac{R^{2}}{2(\kappa+1)}\left(\|\boldsymbol{t}\|^{2}+\kappa\left(\boldsymbol{\mu}^{\prime} \boldsymbol{t}\right)^{2}\right)\right\} \\
\times \frac{\kappa}{\sinh \kappa}\left\{\begin{array}{cc}
\frac{\sin \sqrt{R^{2}\|t\|^{2}-\kappa^{2}}}{\sqrt{R^{2}\|t\| \|^{2}-\kappa^{2}}} & , \boldsymbol{t} \in \Omega, \\
\frac{\alpha \sinh \alpha \cos \beta+\beta \cosh \alpha \sin \beta}{\alpha^{2}+\beta^{2}} & , \boldsymbol{t} \notin \Omega,
\end{array}\right.
\end{gathered}
$$

with $\alpha=\sqrt{(\operatorname{Re} z+|z|) / 2}, \beta=\operatorname{Im} z / \sqrt{2(\operatorname{Re} z+|z|)}$, $z=\kappa^{2}-R^{2}\|\boldsymbol{t}\|^{2}+2 i \kappa R \boldsymbol{\mu}^{\prime} \boldsymbol{t}$ and $\Omega=\left\{\boldsymbol{t} \in \mathbb{R}^{3}\right.$ s.t. $\|\boldsymbol{t}\| \geq$ $\kappa / R$ and $\boldsymbol{t} \perp \boldsymbol{\mu}\}$, if $\kappa>0$, or $\Omega=\mathbb{R}^{3}$, if $\kappa=0$.

Given a set of DW images, we estimate the parameters that define the EAP in Eq.(5) by a least squares fitting of the raw DW intensities to the theoretical ones given by Eq.(6). This is performed using the derivative-free NEWUOA optimization algorithm [10].

Remarks. When all the $\kappa_{i}$ 's go to zero at the same rate, the theoretical DW intensity is modeled as in DTI for low $b$ values. When all the $\kappa_{i}$ 's go to infinity at the same rate and the transverse diffusivity goes to zero, the theoretical DW intensity is modeled as in [11], where a spherical deconvolution approach using Watson pdfs is employed to describe the theoretical DW intensity itself. 


\section{EXPERIMENTAL VALIDATION}

\subsection{Experimental setup}

\subsubsection{Crossing angle resolution of the model}

The crossing angle resolution (CAR) is the minimum detectable angle between two different FOs. We performed its evaluation on the 2 -fiber model $(M=2)$ using synthetic data on a single voxel. We generated the simulated data sets according to the spherical deconvolution method proposed in [12]. They simulate multiple FOs assuming that the fiber orientation distribution function is a sum of equally weighted delta functions. They perform its convolution with the kernel proposed in [13], which models restricted diffusion within a cylindrical fiber of radius $\rho=5 \mu \mathrm{m}$ and length $L=5 \mathrm{~mm}$. We chose this method because (i) they implemented it on-line, as part of the fanDTasia toolbox ${ }^{3}$, (ii) this method has been used in more than 20 papers and (iii) the spherical deconvolution in its discrete version is close to multi-compartment models.

We simulated five data sets of DW images with $b=1500$ $\mathrm{s} / \mathrm{mm}^{2}$ and respectively $n_{g}=15,30,41,64$ and 200 encoding gradient directions uniformly distributed on the hemisphere. For a given $n_{g}$, we generated several configurations of the FOs and we corrupted each data set with twelve increasing noise levels ranging from a signal-to-noise ratio (SNR) of 60 $\mathrm{dB}$ to $0 \mathrm{~dB}$. The configurations of FOs were defined as follows. The first fiber was set to five different values $(\theta, \phi)$, with $\theta=90^{\circ}$ and $\phi=0^{\circ}, 30^{\circ}, 45^{\circ}, 60^{\circ}, 90^{\circ}$. The second fiber was set to 25 different values $(\theta, \phi+\Delta \phi)$ for each value of the first fiber, with $\Delta \phi$ going from $90^{\circ}$ to $60^{\circ}$ by $10^{\circ}$ steps, from $60^{\circ}$ to $30^{\circ}$ by $5^{\circ}$ steps and from $30^{\circ}$ to $0^{\circ}$ by $2^{\circ}$ steps.

The simulated data sets with ground truth crossing angle of $0^{\circ}$ are single fiber cases. The upper bound of the $95 \%$ confidence interval of the estimated crossing angle, referred to as the $\mathbf{9 5 \%}$ confidence angle, is thus a fair approximation of the CAR since any voxel in which the estimated crossing angle drops below this value has a significant probability to contain only one single fiber. We thus resampled each data set 100 times and computed the $95 \%$ confidence angle using the percentile method. In order to minimize the effect of the sensitivity of the NEWUAO algorithm to the initialization, we define the CAR as the minimum $95 \%$ confidence angle over the different fiber configurations that we simulated.

\subsubsection{Real data acquisition}

We aim at demonstrating that our model permits to estimate crossing fibers from LARD images with low spatial resolution. To this end, we scanned a healthy adult male on a 3T Achieva Philips MRI Scanner with an 8-channel head coil, $\mathrm{TR}=10000 \mathrm{~ms}, \mathrm{TE}=64 \mathrm{~ms}, \tau=22.1 \mathrm{~ms}, b=800 \mathrm{~s} / \mathrm{mm}^{2}$, $n_{g}=15$ and $2 \times 2 \times 2 \mathrm{~mm}^{3}$ voxels. This set of DW images represents a typical case of LARD images with low spatial

\footnotetext{
${ }^{3}$ http://www.cise.ufl.edu/ abarmpou/lab/DWMRI.simulator.php
}

resolution. We first denoised the raw DW intensities using the Rician-adapted Non-Local Means filter [14] available on-line ${ }^{4}$. Then, we performed the estimation of the EAP as described in Section 2.2. The denoising step allows us to consider that the mean of the denoised raw DW intensity matches the corresponding theoretical one, which legitimates the estimation procedure described in Section 2.2.

\subsection{Results}

\subsubsection{Estimation of the CAR of the model}

Figure 1 shows that the CAR of our model improves as SNR increases and as the number $n_{g}$ of encoding gradient directions increases. Even in absence of noise, the model cannot distinguish two FOs that are separated by an angle smaller than $1.4^{\circ}$ (for a clinical value of $n_{g}=30$ ). Also, for low SNRs, increasing drastically $n_{g}$ does not seem to improve significantly the CAR. Last, we read in Fig.1 that, for standard clinical dMRI data sets $\left(\mathrm{SNR}=20 \mathrm{~dB}\right.$ and $\left.n_{g}=30\right)$, the CAR of our model is about $30^{\circ}$.

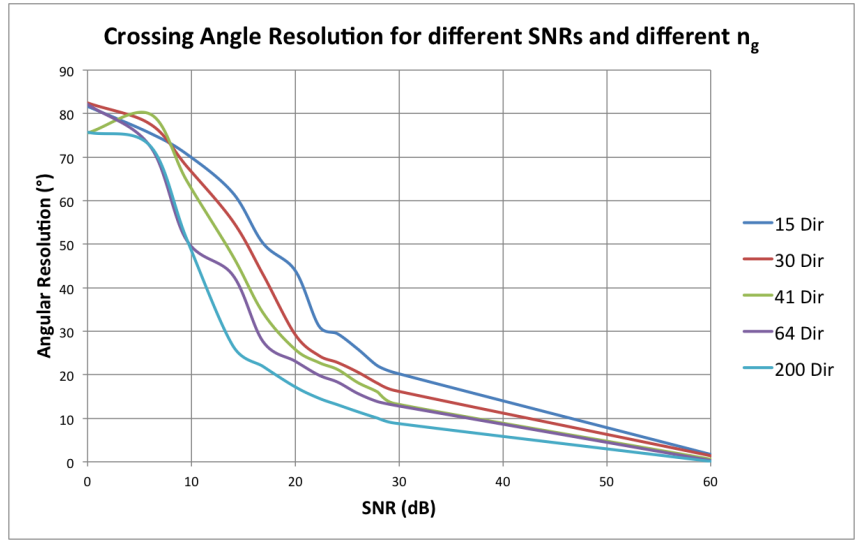

Fig. 1. Crossing angle resolution in ${ }^{\circ}$ of our model (y-axis) for various SNRs in $\mathrm{dB}$ (x-axis). Each curve corresponds to a specific number of encoding gradient directions (cf. legend).

\subsubsection{Real data experiments}

We propose the cone glyph to visualize the parameters that define the EAP in Eq.(5). In each voxel of the image, we display $M$ cones, where $M$ is the number of fiber compartments that we included in the model. The axis of each cone lies on the estimated $\mathrm{FO} \pm \boldsymbol{\mu}$ in the corresponding compartment. The radius of the cone is inversely proportional to the measure of anisotropy $\kappa+1$ (meaning that, the more anisotropic the compartment, the thinner its corresponding cone) and the height of the cone is proportional to the mean squared radial displacement $(\kappa+1) \lambda$ (meaning that the longer water molecules diffuse in the compartment, the longer its corresponding cone). A line thus represent a trustworthy estimated

\footnotetext{
${ }^{4}$ https://www.irisa.fr/visages/benchmarks/
} 
FO while a disk indicates the contrary. Figure 2 shows that our model is capable of accurately estimating crossing fibers from LARD images with low spatial resolution.

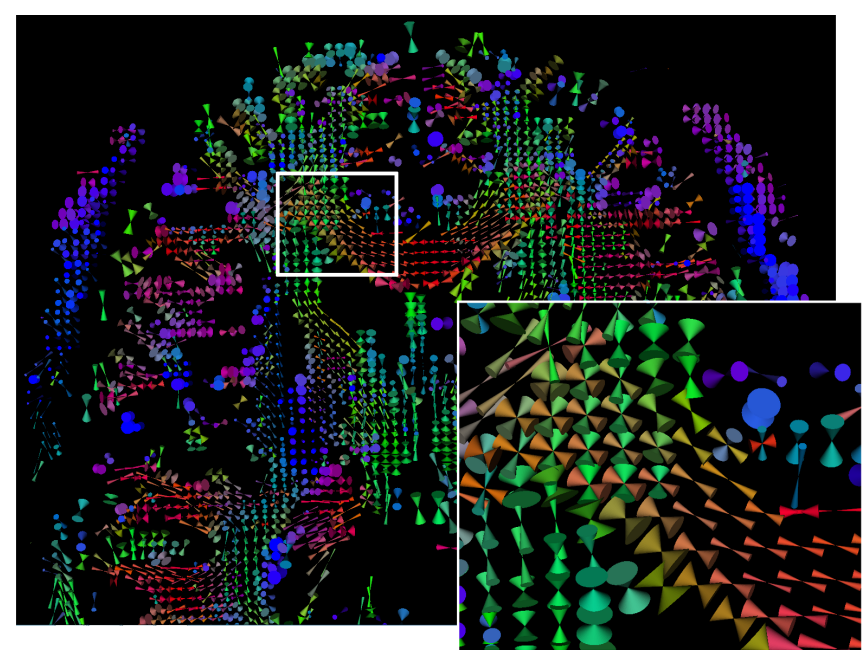

Fig. 2. Cone visualization of the EAP on the 42-th axial slice of the subject's brain. Zoom on an extremity of the corpus callosum known to contain crossing fibers. Colors encode the FO: left/right (red), front/back (green), top/bottom (blue).

\section{DISCUSSION \& PERSPECTIVES}

In this paper, we proposed a new parametric mixture model for the EAP based on non-Gaussian pdfs. It can be estimated from LARD images with low spatial resolution ( $b=1500 \mathrm{~s} / \mathrm{mm}^{2}, n_{g}=30,2 \times 2 \times 2 \mathrm{~mm}^{3}$ voxels). The corresponding CAR of about $30^{\circ}$ outperforms the CAR obtained with the current standard method Q-Ball Imaging [15] for the estimation of crossing fibers, which is around $60^{\circ}$ at a higher angular resolution $\left(n_{g}=81\right)$. Since our model is adapted to clinical brain imaging, it can be used to re-analyze all the DW data sets acquired over the past years. Quantities akin to the fractional anisotropy (FA) and the mean diffusivity (MD) [16] can also be derived from the Gaussian part of our model and by analogy with DTI: $\mathrm{FA}=\kappa\left((\kappa+1)^{2}+2\right)^{-1 / 2}$ and $\mathrm{MD}=(1+\kappa / 3) R^{2}(\kappa+1)^{-1}$.

Some complementary analyses however remain to be carried out and will be the object of a future work to get: (i) a deeper comparison with the most popular state-of-theart methods, (ii) some statistical assessment of the abovementioned versions of FA and MD, and (iii) a model selection procedure for the choice of the number $M$ of fiber compartments.

\section{REFERENCES}

[1] P.J. Basser, J. Mattiello, and D. Le Bihan, "MR diffusion tensor spectroscopy and imaging," Biophys. J., vol. 66, no. 1, pp. 259-67, 1994.
[2] D.S. Tuch et al., "High angular resolution diffusion imaging reveals intravoxel white matter fiber heterogeneity," Magn. Reson. Med., vol. 48, pp. 577-82, 2002.

[3] V.J. Wedeen et al., "Mapping complex tissue architecture with diffusion spectrum magnetic resonance imaging," Magn. Reson. Med., vol. 54, pp. 1377-86, 2005.

[4] Y.C. Wu and A.L. Alexander, "Hybrid diffusion imaging," Neuroimage, vol. 36, no. 3, pp. 617-29, 2007.

[5] M. Descoteaux et al., "Multiple q-shell diffusion propagator imaging," Med. Image Anal., vol. 15, pp. 603-21, 2011.

[6] S. Peled et al., "Geometrically constrained two-tensor model for crossing tracts in DWI," Magn. Reson. Imaging, vol. 24, no. 9, pp. 1263-70, 2006.

[7] E. Kaden, T.R. Knösche, and A. Anwander, "Parametric spherical deconvolution: inferring anatomical connectivity using diffusion MR imaging," Neuroimage, vol. 37, no. 2, pp. 474-88, 2007.

[8] P.T. Callaghan, Principles of Nuclear Magnetic Resonance microscopy, Oxford University Press, 1991.

[9] A. Stamm, P. Pérez, and C. Barillot, "Diffusion Directions Imaging," Tech. Rep., INRIA, 2011.

[10] M. Powell, "The NEWUOA software for unconstrained optimization without derivatives," in Large-scale nonlinear optimization, vol. 83 of Nonconvex Optim. Appl., pp. 255-97. Springer, 2006.

[11] J.G. Malcolm et al., "A filtered approach to neural tractography using the Watson directional function," Med. Image Anal., vol. 14, no. 1, pp. 58-69, 2010.

[12] A. Barmpoutis, B. Jian, and B.C. Vemuri, "Adaptive kernels for multi-fiber reconstruction," in IPMI, 2009, vol. 21 , pp. $338-49$.

[13] O. Söderman and B. Jönsson, "Restricted diffusion in cylindrical geometry," J. Magn. Reson., Ser. A, vol. 117, no. 1, pp. 94-7, 1995.

[14] N. Wiest-Daesslé et al., "Rician noise removal by nonlocal means filtering for low signal-to-noise ratio MRI: applications to DT-MRI," in MICCAI, 2008, pp. 171-9.

[15] M. Descoteaux et al., "Regularized, fast and robust analytical Q-Ball Imaging," Magn. Reson. Med., vol. 58, pp. 497-510, 2007.

[16] P.J. Basser and C. Pierpaoli, "Microstructural and physiological features of tissues elucidated by quantitative Diffusion Tensor MRI," J. Magn. Reson., Ser. B, vol. 111, pp. 209-19, 1996. 